\title{
PARAMETRIC SYNTHESIS OF STATISTICAL COMMUNICATION NETS*
}

\author{
BY \\ H. FRANK (University of California, Berkeley) \\ AND \\ S. L. HAKIMI (Northwestern University)
}

\begin{abstract}
The traffic within the branches of a communication network is often a random variable with an approximate multivariate normal distribution. The analysis of such systems has been discussed in a previous paper. In this paper, we consider the optimum synthesis problem. Nonlinear and linear programming is used to guarantee that a given flow rate probability between a specified pair of terminals is achieved. In particular, a Uniformly Most Powerful Statistical Test provides the basis for an optimum synthesis procedure that appears to be very efficient. The procedure is formulated as a concave program with quadratic and linear constraints.
\end{abstract}

1. Introduction. The existing traffic within the branches $b_{1}, \cdots, b_{b}$ of a $v$ vertex communication network $G$ is usually random. In this case, one may be asked to find the probability that a flow rate $F_{i j}$ of $R$ or more units can be established between a specified pair of terminals $v_{i}$ and $v_{i}$. If this probability is too small, it will be necessary to increase the capacities of the network branches, with minimum cost, until a given probability level is reached.

The analysis problem of finding Prob $\left\{F_{i i} \geq R\right\}$ has been discussed previously [1]-[3]. A general method of analysis requiring knowledge of the joint probability density of the network flows has been presented [1], while a statistical procedure may be used if the probability distributions of the flows are unknown [2]. For this case, an optimum synthesis technique, based on linear programming with some integer variables, can also be given [2]. Often, the traffic may be assumed to have a multivariate normal distribution. Then, Prob $\left\{F_{i j} \geq R\right\}$ may be found by using the methods presented in our earlier paper [3].

In this paper, we investigate the optimum synthesis of statistical communication nets when the probability distribution of the existing network traffic may be approximated by a nonsingular $b$-dimensional normal distribution. The results of this paper depend heavily on the analysis of this class of nets already discussed [3].

II. Synthesis procedures based on multidimensional integrals. We have seen that $\operatorname{Prob}\left\{F_{i i} \geq R\right\}$ can be written as

$$
\begin{gathered}
\operatorname{Prob}\left\{F_{i i} \geq R\right\}=\int \phi\left(y_{1}\right) d y_{1} \int \phi\left(y_{2}\right) d y_{2} \cdots \int \phi\left(y_{r}\right) d y_{r}, \\
\sum_{j=1}^{r} s_{i j} y_{j} \geq k_{i} \quad(j=1, \cdots, q),
\end{gathered}
$$

*Received September 26, 1966. This work was supported in part by the U. S. Army Research Office, Grant No. DA-ARO-D-31-124-G576 and in part by the U. S. Air Force Office of Scientific Information. The notation used in this paper is identical to that used in our previous work [1]-[3]. 
where $\phi(y)$ is the standard normal density function, and $s_{i j}(j=1, \cdots, r ; i=1, \cdots, q)$ is a known constant. This expression is complicated but could be evaluated by means of a digital computer. Let us begin with the simplest form of the synthesis problem. We are allowed to modify only one branch of the network. Assuming equal costs, we must find the "best" branch to modify. (By "best," we mean the branch which gives the required improvement with minimum cost.)

Suppose that we increase the capacity of branch $b_{k}$ from $c_{0 k}$ to $c_{0 k}+\Delta c_{k}$. It should be clear that Prob $\left\{F_{i j} \geq R\right\}$ is a nondecreasing function of $\Delta c_{k}$. Let the cut-sets of $G$ be renumbered so that $b_{k} \in A_{i}, i=1,2, \cdots, t$ and $b_{k} \notin A_{i}, i=t+1, \cdots, q$. Then

$$
\text { Prob }\left\{F_{i i} \geq R\right\}=\operatorname{Prob}\left\{\min _{1 \leq i \leq t}\left|A_{i}\right| \geq R-\Delta c_{k}, \min _{t+1 \leq i \leq q}\left|A_{i}\right| \geq R\right\},
$$

and an upper bound to this probability is given by

$$
\text { Prob }\left\{F_{i i} \geq R\right\} \leq \operatorname{Prob}\left\{\min _{t+1 \leq i \leq q}\left|A_{i}\right| \geq R\right\} .
$$

Now the right-hand side of Eq. (3) is the probability that, in the graph $G_{k}$, obtained from $G$ by shorting (i.e., replacing $b_{k}$ by a branch with infinite capacity) branch $b_{k}$, the maximum flow $F_{i j}$ between vertices $v_{i}$ and $v_{i}$ is at least $R$.

Under our normality assumption, equality in Eq. (3) can only be attained by increasing $\Delta c_{k}$ to infinity. Then the values of cut-sets $\left|A_{1}\right|, \cdots,\left|A_{t}\right|$ will always be at least $R$. Equality can never be attained for any finite value of $\Delta c_{k}$ because the probability distribution of $\left|A_{i}\right|(i \leq t)$ is positive over the entire real line. However, in reality, we are dealing with bounded distributions. That is, if $F_{j}$ is the random flow in $b_{i}$, there is a natural bound $f_{i_{\max }}$ to the magnitude of $F_{i}$ (i.e., Prob $\left\{F_{i}>f_{j \max }\right\}=0$ ). For example, if $G$ is a telephone network, the flow in $b_{i}$ could never exceed the number of people on earth (assuming that only people use telephones). This means that there is a finite $\Delta c_{k}$ such that an increase in the capacity of $b_{k}$ beyond this value will not result in a corresponding increase in Prob $\left\{F_{i i} \geq R\right\}$. In fact,

$$
\text { Prob }\left\{F_{i i} \geq R\right\}=\operatorname{Prob}\left\{\min _{t+1 \leq i \leq q}\left|A_{i}\right| \geq R\right\}
$$

if and only if

$$
\Delta c_{k} \geq R+\sum_{i=1}^{b} b_{i i} f_{j \max }-\sum_{j=1}^{b} b_{i i} c_{0 i}, \quad i=1, \cdots, t .
$$

Thus, we could find the (approximate) maximum flow rate probability obtainable by increasing the capacity of a single branch $b_{k}$ by finding Prob $\left\{F_{i i}^{k} \geq R\right\}$ for the graph $G_{k}$. Therefore, the problem of increasing Prob $\left\{F_{i i} \geq R\right\}$ by increasing the capacity $c_{k}=$ $c_{0 k}+\Delta c_{k}$ is routine and depends only on techniques for evaluating the integrals given in Eq. (1). Moreover, we do not increase the complexity of the problem if we are interested in uniformly increasing the capacities of a subset (or of all) of the branches of $G$. Graphs with uniform branch capacities are of special interest in the theory of communication nets [4]. For this class of graphs, synthesis techniques are conceptually simple.

In Sec. II of [3], we gave bounds for Prob $\left\{F_{i j} \geq R\right\}$. These bounds are

$$
\begin{aligned}
\int_{-\infty}^{\infty}\left[\prod_{i=1}^{a} \Phi\left\{\frac{\left(\rho_{\min }\right)^{1 / 2} y-R_{i}}{\left(1-\rho_{\min }\right)^{1 / 2}}\right\}[\phi(y) d y\right. & \leq \operatorname{Prob}\left\{F_{i i} \geq R\right\} \\
& \leq \int_{-\infty}^{\infty}\left[\prod_{i=1}^{a} \Phi\left\{\frac{\left(\rho_{\max }\right)^{1 / 2} y-R_{i}}{\left(1-\rho_{\max }\right)^{1 / 2}}\right\}\right] \phi(y) d y
\end{aligned}
$$


where $\rho_{\min }$ and $\rho_{\max }$ are the minimum and maximum correlation coefficients of the $\left|A_{i}\right|$, $\Phi$ is the cumulative distribution function of the standard normal variable, and

$$
R_{i}=\frac{R-\sum_{i=1}^{b} b_{i j}\left(c_{i}-\mu_{j}\right)}{\left[\operatorname{Var}\left|A_{i}\right|\right]^{1 / 2}} .
$$

Clearly, if $\rho_{\min }=0$ (i.e., there are at least two disjoint cut-sets), the lower bound in Eq. (6) becomes

$$
\prod_{i=1}^{a}\left[1-\Phi\left(R_{i}\right)\right] \leq \operatorname{Prob}\left\{F_{i j} \geq R\right\}
$$

and, in general,

$$
\prod_{i=1}^{a}\left[1-\Phi\left(R_{i}\right)\right] \leq \int_{-\infty}^{\infty}\left[\prod_{i=1}^{q} \Phi\left\{\frac{\left(\rho_{\min }\right)^{1 / 2} y-R_{i}}{\left(1-\rho_{\min }\right)^{1 / 2}}\right\}\right] \phi(y) d y .
$$

We want to guarantee (with minimum cost) that Prob $\left\{F_{i i} \geq R\right\}$ is at least some probability $p_{0}$. One suboptimum approach to this problem is to guarantee (with minimum cost) that the lower bound in Eq. (7) is at least $p_{0}$. We then have a new problem, which can be stated as

$$
\begin{aligned}
& \text { Find } \Delta c \geq 0 \text {, such that } \\
& \mathbf{h}^{\prime} \Delta \mathbf{c} \text { is minimized }
\end{aligned}
$$

and

$$
\prod_{i=1}^{a}\left[1-\Phi\left(R_{i}\right)\right] \geq p_{0} .
$$

Let $\pi_{i}=1-\Phi\left(R_{i}\right), i=1, \cdots, q$. The constraint of Eq. (9b) can be written as

$$
\prod_{i=1}^{q} \pi_{i} \geq p_{0}
$$

and since $R_{i}=\Phi^{-1}\left(1-\pi_{i}\right)$, we have the relation

$$
R-\sum_{i=1}^{b} b_{i j}\left(c_{i}-\mu_{j}\right)=\beta_{i} \Phi^{-1}\left(1-\pi_{i}\right), \quad i=1, \cdots, q,
$$

where $\beta_{i}^{2}=\operatorname{Var}\left|A_{i}\right|$. Therefore, the entire problem of finding a minimum cost $\Delta \mathrm{c}$ can be written as

Find $\Delta \mathrm{c} \geq 0$ and numbers $\pi_{1}, \cdots, \pi_{q}$ such that

$$
\mathbf{h}^{\prime} \Delta \mathbf{c} \text { is minimized }
$$

and

$$
\begin{gathered}
\prod_{i=1}^{a} \pi_{i} \geq p_{0}, \\
0 \leq \pi_{i} \leq 1, \quad i=1, \cdots, q, \\
\sum_{i=1}^{b} b_{i j}\left(c_{0 i}+\Delta c_{i}-\mu_{i}\right)+\beta_{i} \Phi^{-1}\left(1-\pi_{i}\right)-R=0, \quad i=1, \cdots, q .
\end{gathered}
$$


Furthermore, if $x \geq 0$, it is possible to show that $\Phi(x)$ is concave and for $x \leq 0, \Phi(x)$ is convex. Hence for $1 \geq \pi_{i} \geq \frac{1}{2}, \Phi^{-1}\left(1-\pi_{i}\right)$ is concave and for $0 \leq \pi_{i} \leq \frac{1}{2}, \Phi^{-1}\left(1-\pi_{i}\right)$ is convex. It is easily seen that the set of $\pi_{i}$ for which $\prod_{i=1}^{i} \pi_{i} \geq p_{0}$, is a bounded convex set. Hence, if $p_{0} \geq\left(\frac{1}{2}\right)^{a}, \pi_{i} \geq \frac{1}{2}$ and we have a concave programming problem [5] of the form

$$
\text { Find } \Delta \mathrm{c} \geq \mathbf{0} \text { and } \pi_{1}, \cdots, \pi_{q} \text { such that }
$$

$$
\mathbf{h}^{\prime} \Delta \mathbf{c} \text { is minimized }
$$

subject to

$$
\begin{gathered}
\prod_{i=1}^{q} \pi_{i} \geq p_{0}, \\
\frac{1}{2} \leq \pi_{i} \leq 1, \quad i=1, \cdots, q, \\
\mathrm{~d}_{i}^{\prime} \Delta \mathrm{c}+\beta_{i} g\left(\pi_{i}\right)+k_{i}=0, \quad i=1, \cdots, q,
\end{gathered}
$$

where $\mathrm{d}_{i}^{\prime}=\left(b_{i 1}, b_{i 2}, \cdots, b_{i b}\right), k_{i}=\mathrm{d}_{i}^{\prime}\left(\mathbf{c}_{0}-\mathfrak{u}\right)-R$ and $g\left(\pi_{i}\right)$ is a concave function of $\pi_{i}$. This problem can be written as a concave program with separable constraints [5], if we introduce a new variable $y$ and reformulate the problem as

Find $\Delta \mathrm{c} \geq 0, \pi_{1}, \cdots, \pi_{q}$ and $y$ such that

$$
\mathbf{h}^{\prime} \Delta \mathbf{c} \text { is minimized }
$$

and

$$
\begin{gathered}
y \geq p_{0} \\
\frac{1}{2} \leq \pi_{i} \leq 1, \quad i=1, \cdots, q, \\
\mathbf{d}_{i}^{\prime} \Delta \mathbf{c}+\beta_{i} g\left(\pi_{i}\right)+k_{i}=0, \quad i=1, \cdots, q, \\
\ln y=\sum_{i=1}^{q} \ln \pi_{i} .
\end{gathered}
$$

This problem could readily be solved with existing nonlinear methods. The only drawback is that the number $q$ could be extremely large and thus the program could have a large number of constraints. (For example, a graph with 50 vertices could have millions of cut-sets.)

Suppose $\rho_{\min }>0$. We will now show how an optimization procedure might be based on the lower bound given in Eq. (6). Unless the $R_{i}$ appearing in this bound are negative numbers, it can be seen that Prob $\left\{F_{i i} \geq R\right\}$ is small. Let $R_{i}^{+}=-R_{i}$, and let $R^{+}=$ $\min \left(R_{1}^{+}, \cdots, R_{q}^{+}\right)$. Then, if $a_{1}=\left(\rho_{\min }\right)^{1 / 2} /\left(1-\rho_{\min }\right)^{1 / 2}, a_{2}=1 /\left(1-\rho_{\min }\right)^{1 / 2}$, and $a=a_{2} / a_{1}$

$$
\begin{aligned}
\operatorname{Prob}\left\{F_{i i} \geq R\right\} & \geq \int_{-\infty}^{\infty}\left[\prod_{i=1}^{a} \Phi\left(a_{1} y+a_{2} R_{i}^{+}\right)\right] \phi(y) d y \\
& \geq \int_{-\infty}^{\infty} \Phi^{q}\left(a_{1} y+a_{2} R^{+}\right) \phi(y) d y .
\end{aligned}
$$

Also, we may write

$$
\begin{aligned}
\int_{-\infty}^{\infty} \Phi^{a}\left(a_{1} y+\right. & \left.a_{2} R^{+}\right) \phi(y) d y \\
& =\int_{-\infty}^{-a R^{+}} \Phi^{a}\left(a_{1} y+a_{2} R^{+}\right) \phi(y) d y+\int_{-a R^{+}}^{\infty} \Phi^{a}\left(a_{1} y+a_{2} R^{+}\right) \phi(y) d y,
\end{aligned}
$$


and since $\Phi(0)=\frac{1}{2} \geq \Phi\left(a_{1} y+a_{2} R^{+}\right)$, for all $y$ in the interval $-\infty<y \leq-a R^{+}$,

$$
\int_{-\infty}^{-a R^{+}} \Phi^{a}\left(a_{1} y+a_{2} R^{+}\right) \phi(y) d y \leq\left(\frac{1}{2}\right)^{a} \int_{-\infty}^{-a R^{+}} \phi(y) d y \leq\left(\frac{1}{2}\right)^{a+1} .
$$

We can therefore neglect this term and use the approximation

$$
\int_{-\infty}^{\infty} \Phi^{a}\left(a_{1} y+a_{2} R^{+}\right) \phi(y) d y \doteq \int_{-a^{+}}^{\infty} \Phi^{a}\left(a_{1} y+a_{2} R^{+}\right) \phi(y) d y .
$$

Let $z=a_{1} y+a_{2} R^{+}$. For $z \geq 0$, we can substitute the approximate expression [6]

into Eq. (18) to obtain

$$
\Phi(z) \doteq \frac{1}{2}\left[1+\left(1-e^{-2 z^{2 / \pi}}\right)^{1 / 2}\right]
$$

$\int_{-\infty}^{\infty} \Phi^{a}\left(a_{1} y+a_{2} R^{+}\right) \phi(y) d y \doteq \frac{1}{a_{1}}\left(\frac{1}{2}\right)^{a} \int_{0}^{\infty}\left[1+\left(1-e^{-2 z^{2 / \pi}}\right)^{1 / 2}\right]^{a} \phi\left(\frac{z-a_{2} R^{+}}{a_{1}}\right) d z$.

Expanding $\Phi^{q}(z)$ as given by Eq. (19) into a binomial series, we obtain

$$
\Phi^{q}(z) \doteq\left(\frac{1}{2}\right)^{a} \sum_{k=0}^{q}\left(\begin{array}{l}
q \\
k
\end{array}\right)\left(1-e^{-2 z^{2 / \pi}}\right)^{k / 2},
$$

and we may further expand the factor

$$
\left(1-e^{-2 z^{2 / \pi}}\right)^{k / 2}
$$

into another binomial series. The integral on the right-hand side of Eq. (20) may then be written as

$$
\begin{aligned}
\sum_{k=0 ; k \text { even }}^{e} \frac{\left(\begin{array}{l}
q \\
k
\end{array}\right)\left(\frac{1}{2}\right)^{a}}{a_{1}} \sum_{i=0}^{k / 2}(-1)^{i}\left(\begin{array}{c}
k / 2 \\
j
\end{array}\right) I_{i_{1}}\left(R^{+}\right) \\
+\sum_{k=1: k \text { odd }}^{a} \frac{\left(\begin{array}{l}
q \\
k
\end{array}\right)\left(\frac{1}{2}\right)^{a}}{a_{1}} \sum_{j=0}^{(k-1) / 2}(-1)^{j}\left(\begin{array}{c}
(k-1) / 2 \\
j
\end{array}\right) I_{i_{\mathbf{3}}}\left(R^{+}\right),
\end{aligned}
$$

where

and

$$
I_{i_{1}}\left(R^{+}\right)=\int_{0}^{\infty} e^{-2 i z^{2} / \pi} \phi\left(\frac{z-a_{2} R^{+}}{a_{1}}\right) d z
$$

$$
I_{i_{3}}\left(R^{+}\right)=\int_{0}^{\infty}\left(1-e^{-2 z^{2 / \pi}}\right)^{1 / 2} e^{-2 i z^{2 / \pi}} \phi\left(\frac{z-a_{2} R^{+}}{a_{1}}\right) d z .
$$

For a given value of $R^{+}, I_{i_{1}}$ and $I_{i_{2}}$ could be evaluated with numerical techniques. Furthermore, we know that $\int \Phi^{q}\left(a_{1} y+a_{2} R^{+}\right) \phi(y) d y$ is an increasing function of $R^{+}$. Let $R_{0}^{+}$be the smallest value of $R^{+}$for which the number given in expression (22) is $p_{0}$ After we have found $R_{0}^{+}$, with numerical techniques, we can solve the suboptimum synthesis problem.

By definition,

$$
R_{i}^{+}=\left[R-\sum_{i=1}^{b} b_{i j}\left(c_{i}-\mu_{i}\right)\right] / \beta_{i}, \quad i=1, \cdots, q
$$

and $R^{+}=\min \left(R_{1}^{+}, \cdots, R_{q}^{+}\right)$. Given $R_{0}^{+}$, we want to find a capacity vector $\Delta \mathrm{c}$ such that $R_{0}^{+}=\min \left(R_{1}^{+}, \cdots, R_{q}^{+}\right)$and $\mathbf{h}^{\prime} \Delta \mathrm{c}$ is minimized. We can do this with the following 
linear program.

and

Find $\Delta \mathrm{c} \geq 0$ such that

$$
\mathbf{h}^{\prime} \Delta \mathbf{c} \text { is minimized }
$$

$$
\sum_{j=1}^{b} b_{i j}\left(c_{0 j}+\Delta c_{j}-\mu_{j}\right) \geq R-\beta_{i} R_{0}^{+}, \quad i=1, \cdots, q .
$$

If $\beta_{1}=\beta_{2}=\cdots=\beta_{a}$, the above linear program becomes exceptionally manageable. In this case, the constraint becomes

$$
B\left(c_{0}+\Delta \mathbf{c}-\mathfrak{u}\right) \geq\left(\begin{array}{c}
k \\
k \\
\vdots \\
k
\end{array}\right)
$$

where $k=R-\beta_{1} R_{0}^{+}$. This problem is discussed by Deo and Hakimi [7] and may be solved with the linear program:

Find $\mathrm{X}_{1} \geq 0, \mathrm{X}_{2} \geq 0, X_{m+1} \geq 0$ and $\Delta \mathrm{c} \geq 0$ such that

and

$$
\mathbf{h}^{\prime} \Delta \mathbf{c} \text { is minimized, }
$$

$$
\begin{gathered}
U^{*}\left[\begin{array}{c}
\mathrm{X}_{1}-\mathrm{X}_{2} \\
X_{m+1}
\end{array}\right]=0, \\
\mathbf{X}_{1}+\mathrm{X}_{2} \leq \mathbf{c}_{0}-\boldsymbol{u}+\Delta \mathbf{c}, \\
X_{m+1}=k,
\end{gathered}
$$

where $\mathbf{X}_{1}$ and $\mathbf{X}_{2}$ are $b$-dimensional vectors and $U^{*}$ is the incidence matrix of a graph $G^{*}$, obtained from $G$ by arbitrarily orienting the branches of $G$ and then adding a branch $b_{b+1}^{*}$, directed from $v_{i}$ to $v_{i}$.

We can continue in the above vein and formulate other programming problems, based on the constraint Prob $\left\{F_{i j} \geq R\right\} \geq p_{0}$, approximations to this constraint, or other lower bounds. However, at this point, the numerical difficulties that enter the picture make such formulations impractical. To obtain a significant increase in the computational efficiency of our procedures, we must adopt a different approach. One such approach can be based on the empirical observations of Secs. III and IV of [3]. We have shown that Prob $\left\{F_{i i} \geq R\right\}$ can, with reasonable accuracy, be written as

$$
\operatorname{Prob}\left\{F_{i i} \geq R\right\} \doteq 1-\Phi\left(\frac{R-\nu_{1}}{\sigma}\right),
$$

where $\nu_{1}$ and $\sigma^{2}$ are the unknown mean and variance of $\min \left(\left|A_{1}\right|, \cdots,\left|A_{q}\right|\right)$.

The constraint Prob $\left\{F_{i j} \geq R\right\} \geq p_{0}$ is thus (approximately) equivalent to

$$
\begin{gathered}
1-\Phi\left(\frac{R-\nu_{1}}{\sigma}\right) \geq p_{0}, \\
\left(\nu_{1}-R\right) / \sigma \geq \theta_{0} \triangleq-\Phi^{-1}\left(1-p_{0}\right),
\end{gathered}
$$

or

$$
\nu_{1}-\sigma \theta_{0} \geq R
$$


We can compute $\nu_{1}$ and $\sigma$ by using Clark's Method [8], discussed in Sec. IV of [3]. Then the constraint given by Eq. (28c) suggests that this method, in conjunction with a steepest descent technique [5], could be used to attack the synthesis problem. However, we will use a different approach, based on the statistical test given in Sec. V of [3].

III. Parametric statistical synthesis. Assume that a set of branch flow observations $\{\mathcal{F}(k) ; k=1, \cdots, n\}$ is available. As in [2] and [3], $F(1), \cdots, \mathcal{F}(n)$ are identically and independently distributed random variables. Then, if the maximum flow $F_{i i}$ is normally distributed, a Uniformly Most Powerful Invariant level $\alpha$ test for testing

against

$$
H_{1}: p=\operatorname{Prob}\left\{F_{i i} \geq R\right\} \geq p_{0}
$$

$$
H_{2}: p<p_{0}
$$

is

$$
\text { Reject } H_{1} \text { if } t(\{F\})<K(n(n-1))^{-1} \Delta K^{\prime}
$$

and

$$
\text { Accept } H_{1} \text { if } t(\{\mathscr{F}\}) \geq K(n(n-1))^{-1}=K^{\prime}
$$

where

$$
\begin{gathered}
t(\{\mathfrak{F}\})=\frac{(\bar{m}-R)}{\left[\sum_{k=1}^{n}\left(m_{k}-\bar{m}\right)^{2}\right]^{1 / 2}}, \\
m_{k}=\min _{1 \leq i \leq a} \sum_{j=1}^{b} b_{i j}\left(c_{i}-f_{i}(k)\right), \\
\bar{m}=\frac{1}{n} \sum_{k=1}^{n} m_{k},
\end{gathered}
$$

and $K$ is a constant determined by

$$
\begin{array}{r}
\int_{-\infty}^{K}\left[\int_{0}^{\infty} w^{(n-2) / 2} \exp -\left(\frac{w}{2}\right) \exp \left(t\left(\frac{w}{n-1}\right)^{1 / 2}+n^{1 / 2} \Phi^{-1}\left(1-p_{0}\right)\right)^{2} d w\right] d t \\
=\alpha\left[2^{n / 2} \Gamma\left(\frac{n-1}{2}\right)(\pi(n-1))^{1 / 2}\right] .
\end{array}
$$

Suppose that on the basis of the observed data, we reject $H_{1}$. Then the probability that this is an incorrect decision is no greater than $\alpha$. Thus, if $\alpha$ is small, we must increase the branch capacities of $G$. We have already given a synthesis procedure when $\mathcal{F}(k)$ and $F_{i}$ have unknown probability distributions [2]. We can use the same philosophy of synthesis if the probability distribution of maximum flow is known. We simply increase the capacities of the branches of $G$ until $t(\{\mathcal{F}\})>K^{\prime}$; in other words, until $H_{1}$ is accepted. If this procedure is completed with minimum cost, we have optimally improved the net. Moreover, the probability that Prob $\left\{F_{i i} \geq R\right\}<p_{0}$ is minimum (among all invariant procedures).

Hypothesis $H_{1}$ will be accepted if

$$
\frac{\bar{m}-R}{\left[\sum_{k=1}^{n}\left(m_{k}-\bar{m}\right)^{2}\right]^{1 / 2}} \geq K^{\prime} .
$$


Since

$$
m_{k}=\min _{1 \leq i \leq a} \sum_{j=1}^{b} b_{i j}\left(c_{0 i}+\Delta c_{i}-f_{j}(k)\right),
$$

we would like to find values of $\Delta c_{1}, \cdots, \Delta c_{b}$ such that Eq. (31) is satisfied. This constraint is equivalent to the set of constraints

$$
\begin{gathered}
\bar{m}-R-K^{\prime} \hat{z}^{1 / 2} \geq 0 \\
\hat{z}=z-\tilde{z} \\
z-\left(m_{1}, \cdots, m_{n}\right) W\left(m_{1}, \cdots, m_{n}\right)^{\prime}-\tilde{z} \geq 0,
\end{gathered}
$$

where $W$ is an $n-n$ matrix given by

$$
W=\left[\begin{array}{ccccc}
\left(1-\frac{1}{n}\right) & -\frac{1}{n} & -\frac{1}{n} & \cdots & -\frac{1}{n} \\
-\frac{1}{n} & \left(1-\frac{1}{n}\right) & -\frac{1}{n} & \cdots & -\frac{1}{n} \\
-\frac{1}{n} & -\frac{1}{n} & \left(1-\frac{1}{n}\right) \cdots & -\frac{1}{n} \\
\vdots & \vdots & \vdots & \vdots \\
-\frac{1}{n} & -\frac{1}{n} & -\frac{1}{n} & & \left(1-\frac{1}{n}\right)
\end{array}\right]
$$

and $z, \tilde{z}$, and $\hat{z}$ are nonnegative variables.

We can force Eqs. (32a)-(32c) to be satisfied with minimum cost, and at the same time eliminate our dependency on the cut-set matrix $B$, if we use Deo and Hakimi's approach [7], with which Eqs. (26a)-(26d) are written. We again define the graph $G^{*}$, obtained from $G$ by arbitrarily orienting the branches of $G$ and adding a branch directed from $v_{i}$ to $v_{i}$. Then, if $U^{*}$ is the incidence matrix of $G^{*}$, it can be shown that our synthesis problem may be written as

Find $\mathrm{Y} \geq 0, \mathrm{X}_{1 k} \geq 0, \mathrm{X}_{2 k} \geq 0 \quad(k=1, \cdots, n), z \geq 0, \hat{z} \geq 0, \tilde{z} \geq 0$ and $\Delta \mathrm{c} \geq 0$ such that

$$
\mathbf{h}^{\prime} \Delta \mathbf{c} \text { is minimized }
$$

and

$$
\begin{gathered}
U^{*}\left[\begin{array}{c}
\left.\mathbf{X}_{1 k}-\mathbf{X}_{2 k}\right] \\
y_{k}
\end{array}\right]=0, \quad k=1, \cdots, n, \\
\mathbf{X}_{1 k}+\mathbf{X}_{2 k} \leq \mathbf{c}_{0}+\Delta \mathbf{c}-\mathcal{F}(k), \quad k=1, \cdots, n, \\
\frac{1}{n} \sum_{k=1}^{n} y_{k}-R-K^{\prime} \hat{z}^{1 / 2} \geq 0, \\
\hat{z}=z-\tilde{z}, \\
z-\left(y_{1}, \cdots, y_{n}\right) W\left(y_{1}, \cdots, y_{n}\right)^{\prime}-\tilde{z} \geq 0 .
\end{gathered}
$$


Thus, the solution of our problem is in the form of a nonlinear program. It can be shown that for $n>1$, the matrix $W$ is positive semidefinite. This means that the constraint given by Eq. (33f) is concave. The only other nonlinear constraint is the one given by Eq. (33d). This constraint is convex and, for each fixed $\hat{z}$, is linear. The remaining constraints are linear and there are no dependencies on the cut-set matrix of $G$. (The incidence matrix $U^{*}$ contains all necessary information about $G$. Since $U^{*}$ is a $v-b$ matrix, reasonably large nets can be considered.) For each fixed $\hat{z}$, we have an extremely manageable concave programming problem with exactly one nonlinear (quadratic) constraint. Therefore, for each fixed $\hat{z}$ we can use standard concave programming techniques [5] to obtain solutions. Each such solution will force hypothesis $H_{1}$ to be accepted with minimum cost for the given value of $\hat{z}$. Thus, by varying $\hat{z}$ and solving a sequence of such problems, we can find a solution which is arbitrarily close to the optimal solution. Consequently, if we are willing to allow statistical error in our synthesis procedure, we can give an optimum synthesis procedure that is quite practical from a computational point of view.

IV. Conclusions and further remarks. There are other synthesis goals besides minimizing a cost function while achieving a given flow rate probability. For example, we may be allowed to spend a fixed sum of money to improve an existing network. Naturally, we want to buy the maximum possible improvement and so we could attempt to maximize the flow rate probability. If we assume linear cost, we have the new problem

$$
\begin{gathered}
\text { Find } \Delta \mathrm{c} \geq 0 \text { such that } \\
\text { Prob }\left\{F_{i i} \geq R\right\} \text { is maximized }
\end{gathered}
$$

and

$$
\mathrm{h}^{\prime} \Delta \mathrm{c}=Q \quad \text { (a constant). }
$$

We can use the analysis and synthesis techniques that we have already developed to attack this problem. For example, under the normality assumptions of our parametric statistical test, the problem reduces to

$$
\begin{gathered}
\text { Find } \Delta \mathrm{c} \geq 0 \text { such that } \\
\frac{\bar{m}-R}{\left[\sum_{k=1}^{n}\left(m_{k}-\bar{m}\right)^{2}\right]^{1 / 2}} \text { is maximized }
\end{gathered}
$$

and

$$
\mathbf{h}^{\prime} \Delta \mathbf{c}=Q .
$$

In many instances, it is adequate to maximize the expected terminal capacity. Then, if the variance is small, we can expect Prob $\left\{F_{i j} \geq R\right\}$ to be large. Thus, we could consider the problem: maximize $E F_{i j}$, subject to $\mathbf{h}^{\prime} \Delta \mathrm{c}=Q$. In terms of our statistical test, we want to maximize $n \bar{m}=\sum_{k=1}^{n} m_{k}$; consequently, we can solve this problem via linear programming.

Another objective could be minimum variance. We might be willing to accept a lower average maximum flow if we are sure that the actual performance of the system will not deviate far from the average. For the statistical problem, we then minimize the sample variance

$$
\sum_{k=1}^{n}\left(m_{k}-\bar{m}\right)^{2}
$$


We must then solve a quadratic programming problem. (Note that we can also easily constrain the sample mean to be at least some number $R^{*}$ with the constraint $\vec{m} \geq R^{*}$.)

The ideas discussed here could be used to synthesize a communication net, rather than to improve an existing net. To begin the procedure, it would be necessary to estimate the local demands between pairs of stations. Then, a set of branch capacities could be selected so that the local demands are satisfied with a given probability [9]. Then we could begin to consider the terminal capacities between various pairs of stations in the network. To apply our statistical procedures, we could generate the set of flow vectors $\{\mathcal{F}(k)\}$ with Monte Carlo techniques. An important feature of our synthesis procedures is that we do not have to restrict ourselves to improving flow-rate probabilities between single pairs of stations. Instead, we could consider the index I of the communication net [4], defined by

$$
I=\sum_{i=i+1}^{\infty} \sum_{i=1}^{j} F_{i i},
$$

and a possible synthesis goal could be

$$
\text { Prob }\{I \geq k\} \geq p_{0} .
$$

For this problem, the statistical procedures we have given appear quite promising.

\section{APPENDIX}

\section{Illustrative examples}

Example 1. Let $G_{1}$ be the graph shown in Fig. 1. Suppose that the existing branch flows in $G_{1}$ are identically and independently distributed normal variables with mean $\mu_{1}=1$ and variance $\sigma_{i}^{3}=1$. We wish to guarantee that Prob $\left\{F_{12} \geq 3\right\} \geq 0.9$ by increasing (if necessary) the capacities of the branches of $G_{1}$.

Assume that the cost function $\mathbf{h}^{\prime} \Delta \mathbf{c}=\Delta c_{1}+\Delta c_{2}+\Delta c_{3}+\Delta c_{4}+\Delta c_{5}$. Then, in order to guarantee that Prob $\left\{F_{12} \geq 3\right\} \geq 0.9$, we will guarantee that a lower bound to Prob $\left\{F_{12} \geq 3\right\}$ is at least 0.9 . Furthermore, we will do this with minimum cost and will

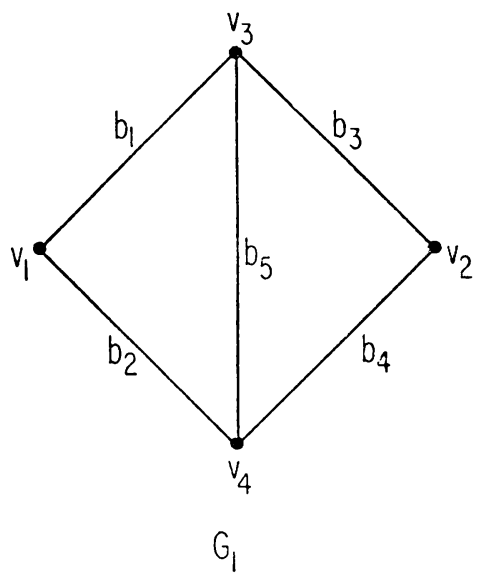

FIGURE 1. 
thus obtain a suboptimum solution to the problem: Find $\Delta \mathrm{c} \geq 0$ such that Prob $\left\{F_{12} \geq 3\right\} \geq 0.9$ and $\mathrm{h}^{\prime} \Delta \mathrm{c}$ is minimized.

From Eq. (7),

where

$$
\prod_{i=1}^{a}\left[1-\Phi\left(R_{i}\right)\right] \leq \operatorname{Prob}\left\{F_{12} \geq 3\right\}
$$

$$
R_{i}=\left[3-\sum_{i=1}^{5} b_{i j}\left(c_{i}-\mu_{j}\right)\right]\left[\operatorname{Var}\left|A_{i}\right|\right]^{1 / 2}
$$

and $A_{1}, \cdots, A_{q}$ are the cut-sets of $G_{1}$. For $G_{1}$ given in Fig. 1, the cut-set matrix is

$$
B=\left[\begin{array}{lllll}
1 & 1 & 0 & 0 & 0 \\
1 & 0 & 0 & 1 & 1 \\
0 & 1 & 1 & 0 & 1 \\
0 & 0 & 1 & 1 & 0
\end{array}\right] .
$$

Also, since $F_{1}, \cdots, F_{5}$ are independent random variables, the variances of $\left|A_{1}\right|,\left|A_{2}\right|$, $\left|A_{3}\right|$, and $\left|A_{4}\right|$ are 2, 3, 3, and 2, respectively. Therefore, from Eqs. (12a)-(12d), the nonlinear program which guarantees that $\prod_{i=1}^{4}\left[1-\Phi\left(R_{i}\right)\right] \geq 0.9$ with minimum cost is:

Find $\Delta \mathrm{c} \geq 0$ and $\pi_{1}, \pi_{2}, \pi_{3}, \pi_{4}$ such that

$$
\begin{aligned}
& \Delta c_{1}+\Delta c_{2}+\Delta c_{3}+\Delta c_{4}+\Delta c_{5} \text { is minimized, } \\
& \pi_{1} \pi_{2} \pi_{3} \pi_{4} \geq 0.9, \\
& 0 \leq \pi_{1} \leq 1 \quad i=1, \cdots, 4 \\
& 2^{1 / 2} \times \Phi^{-1}\left(1-\pi_{1}\right)+1+\Delta c_{1}+\Delta c_{2}=0, \\
& 3^{1 / 2} \times \Phi^{-1}\left(1-\pi_{2}\right)+3+\Delta c_{1}+\Delta c_{4}+\Delta c_{5}=0, \\
& 3^{1 / 2} \times \Phi^{-1}\left(1-\pi_{3}\right)+3+\Delta c_{2}+\Delta c_{3}+\Delta c_{5}=0, \\
& 2^{1 / 2} \times \Phi^{-1}\left(1-\pi_{4}\right)+1+\Delta c_{3}+\Delta c_{4}=0 .
\end{aligned}
$$

This problem can be solved to give $\Delta c_{1} \doteq 1.4, \Delta c_{2}=0, \Delta c_{3} \doteq 1.4, \Delta c_{4}=0$, and $\Delta c_{5}=0$.

Example 2. Consider the graph $G_{2}$ shown in Fig. 2. Suppose we are given the

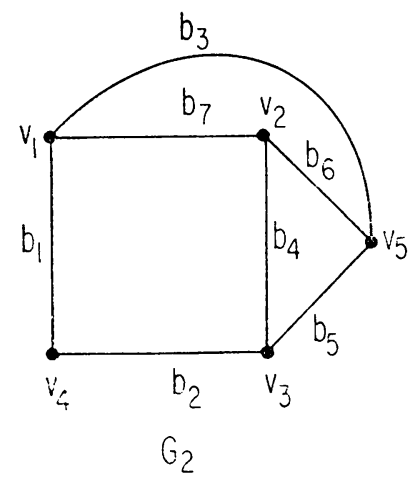

Figure 2 . 
sequence of observations of branch flow $\mathcal{F}(1), \mathcal{F}(2), \mathcal{F}(3), \mathcal{F}(4)$ and $\mathcal{F}(5)$ shown below. We wish to maximize the expected flow rate from vertex $v_{1}$ to vertex $v_{5}$ subject to the constraint that we have a fixed amount $Q$ of capital to spend.

$$
\begin{aligned}
& \mathscr{F}(1)=(76,20,15,7,28,60,47)^{\prime}, \\
& F(2)=(49,31,19,9,31,40,29)^{\prime}, \\
& \mathscr{F}(3)=(83,37,24,11,40,31,36)^{\prime}, \\
& \mathscr{F}(4)=(57,31,17,4,25,45,41)^{\prime}, \\
& \mathscr{F}(5)=(61,25,6,7,17,55,50)^{\prime} .
\end{aligned}
$$

The cost of increasing the capacity of the ith branch by a unit amount is $h_{i}$. Assume that the specified cost vector $\mathbf{h}=\left(h_{1}, \cdots, h_{7}\right)^{\prime}$ is given by $\mathbf{h}=(5,7,10,15,8,6,6)^{\prime}$ and that $Q=500$ dollars are available for the improvement of the network. The initial branch capacity vector is assumed to be $\mathbf{c}_{0}=(100,48,26,13,42,65,52)^{\prime}$.

The first step in the synthesis procedure is to construct the graph $G_{2}^{*}$, obtained from $G_{2}$ by arbitrarily orienting the branches of $G_{2}$ and adding branch $b_{8}$ from $v_{5}$ to $v_{1}$. One possible $G_{2}^{*}$ obtained in this manner is shown in Fig. 3. The incidence matrix $U^{*}$ of $G_{2}^{*}$ is

$$
L^{*}=\left[\begin{array}{rrrrrrrr}
-1 & 0 & 1 & 0 & 0 & 0 & 1 & 0 \\
0 & 0 & 0 & 1 & 0 & 1 & -1 & 0 \\
0 & -1 & 0 & -1 & 1 & 0 & 0 & 0 \\
1 & 1 & 0 & 0 & 0 & 0 & 0 & -1 \\
0 & 0 & -1 & 0 & -1 & -1 & 0 & 1
\end{array}\right]
$$

The problem can now be formulated as the linear program: Find nonnegative vectors $\mathrm{X}_{1 k}, \mathbf{X}_{2 k}$ for $k=1, \cdots, 5$ and nonnegative numbers $y_{1}, \cdots, y_{5}$ and $\Delta c_{1}, \Delta c_{2}, \cdots, \Delta c_{7}$ such that

$$
\sum_{k=1}^{5} y_{k}
$$

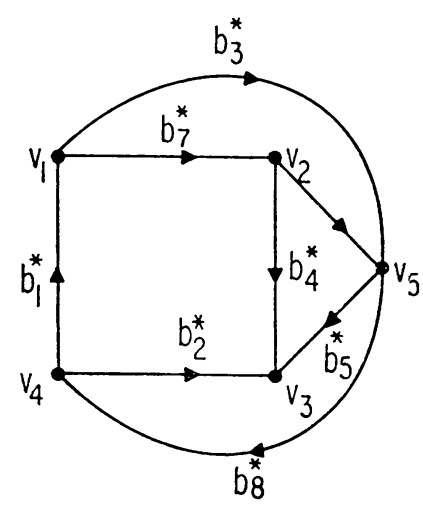

$\mathrm{G}_{2}^{*}$

Figure 3. 
is maximized, subject to

and

$$
\begin{gathered}
U^{*}\left[\begin{array}{c}
\mathbf{X}_{1 k}-\mathbf{X}_{2 k} \\
y_{k}
\end{array}\right]=0, \quad k=1, \cdots, 5, \\
\mathbf{X}_{1 k}+\mathbf{X}_{2 k} \leq \mathrm{c}_{0}+\Delta \mathrm{c}-\mathcal{F}(k), \quad k=1, \cdots, 5,
\end{gathered}
$$

$$
\mathrm{h}^{\prime} \Delta \mathrm{c} \leq 500 \text {. }
$$

The problem is in the standard linear programming format for which computer programs are readily available. The solution of the problem is $\Delta c_{1}=19, \Delta c_{2}=0$, $\Delta c_{3}=33, \Delta c_{4}=0, \Delta c_{5}=7, \Delta c_{6}=0$, and $\Delta c_{7}=3$. Furthermore, it can be shown that the average flow attainable between $v_{1}$ and $v_{5}$ (in excess of the existing branch flows) is 34 before increasing the capacities of the branches of $G$ and is 71 after the branch capacities are increased.

ExAmple 3. Let $G_{1}$ be the graph shown in Fig. 1. Suppose we must guarantee with probability $p_{0}=0.9$ that a flow rate of at least 3 units can be attained between vertices $v_{1}$ and $v_{2}$. Assume that the maximum flow rate $F_{12}$ is a normal random variable and that ten values of the branch flow vector have been observed. On the basis of these flow vectors, we must determine whether or not $\operatorname{Prob}\left\{F_{12} \geq 3\right\} \geq 0.9$. If we conclude that Prob $\left\{F_{12} \geq 3\right\}<0.9$, we must modify the network with minimum cost. Assume that the cost function is $h_{1} \Delta c_{1}+h_{2} \Delta c_{2}+h_{3} \Delta c_{3}+h_{4} \Delta c_{4}+h_{5} \Delta c_{5}$ where the $h_{i}$ are positive constants. Furthermore, assume that the probability of Type I error is specified to be no larger than $\alpha=.1$ and that the observed flow vectors are given by

$$
\begin{aligned}
& \mathcal{F}(1)=\left[\begin{array}{l}
2 \\
2 \\
1 \\
1 \\
2
\end{array}\right] \\
& F(2)=\left[\begin{array}{l}
2 \\
1 \\
3 \\
0 \\
0
\end{array}\right] \\
& \mathfrak{F}(3)=\left[\begin{array}{l}
3 \\
1 \\
1 \\
2 \\
2
\end{array}\right] \\
& \mathcal{F}(4)=\left[\begin{array}{l}
1 \\
1 \\
1 \\
1 \\
1
\end{array}\right] \\
& \mathfrak{F}(5)=\left[\begin{array}{l}
2 \\
1 \\
1 \\
2 \\
2
\end{array}\right] \\
& \mathcal{F}(6)=\left[\begin{array}{l}
1 \\
2 \\
2 \\
1 \\
2
\end{array}\right] \\
& \mathcal{F}(8)=\left[\begin{array}{l}
0 \\
3 \\
0 \\
3 \\
3
\end{array}\right] \\
& F(9)=\left[\begin{array}{l}
3 \\
0 \\
1 \\
2 \\
1
\end{array}\right]
\end{aligned}
$$

The first step in the synthesis procedure is to apply the statistical test given in Eqs. (29) and (30). To apply the test, we must find $K$ as defined in Eq. (30). Rather than directly use the definition of $K$, we will use an approximate method given on page 949 of [6] to find $K$. According to this method, if $\alpha<\frac{1}{2}, K$ corresponds to the smallest root of the quadratic equation

$K^{2}\left[\left(1-\frac{1}{4(n-1)}\right)^{2}-\left(\Phi^{-1}(\alpha)\right)^{2}\right]+2 \delta K\left(1-\frac{1}{4(n-1)}\right)+\delta^{2}-\left(\Phi^{-1}(\alpha)\right)^{2}=0$,

where $\delta=n^{1 / 2} \Phi^{-1}\left(1-p_{0}\right)$. From the preceding equation, $K \doteq 2.55$. 
Now that we have found $K$, we can apply the statistical test. The test rejects the hypothesis $H_{0}$ : Prob $\left\{F_{i j} \geq 3\right\} \geq 0.9$ if

$$
\left(\frac{1}{10} \sum_{k=1}^{10} m_{k}-3\right)\left[\sum_{k=1}^{10} m_{k}^{2}-\frac{1}{10}\left(\sum_{k=1}^{10} m_{k}\right)^{2}\right]^{-1 / 2} \leq K \frac{1}{3(10)^{1 / 2}} .
$$

The maximum flows corresponding to $F(1), \cdots, \mathcal{F}(10)$ can easily be shown to be $m_{1}=2$, $m_{2}=2, m_{3}=2, m_{4}=4, m_{3}=3, m_{6}=3, m_{7}=4, m_{8}=3, m_{9}=3, m_{10}=3$. Therefore, the left-hand side of Eq. (A9) is equal to -0.0407 while the right-hand side is equal to .268. Hence, we reject hypothesis $H_{0}$. (Note that for this example, the sample mean is $\bar{m}=2.9$ and the sample variance is 4.9 . If the sample mean had been larger than 3.593 and the sample variance were no larger than 4.9 , we would accept $H_{0}$ ).

The hypothesis that Prob $\left\{F_{12} \geq 3\right\} \geq 0.9$ has been rejected and so we must increase the capacities of some of the branches in the network. Let $G_{1}^{*}$ be the graph shown in Fig. 4. $G_{1}^{*}$ is obtained from $G_{1}$ by arbitrarily orienting the branches of $G_{1}$ and adding the branch $b_{6}$ directed from $v_{2}$ to $v_{1}$. The incidence matrix $U^{*}$ of $G_{1}^{*}$ is

$$
U^{*}=\left[\begin{array}{rrrrrr}
1 & 1 & 0 & 0 & 0 & -1 \\
0 & 0 & -1 & -1 & 0 & 1 \\
-1 & 0 & 1 & 0 & 1 & 0 \\
0 & -1 & 0 & 1 & -1 & 0
\end{array}\right]
$$

In order to satisfy the constraint given by Eq. (33b), the following equations must hold ( $U^{*}$ has rank 3 and so we can delete one row, say the last row):

$$
\left[\begin{array}{rrrrrr}
1 & 1 & 0 & 0 & 0 & -1 \\
0 & 0 & -1 & -1 & 0 & 1 \\
-1 & 0 & 1 & 0 & 1 & 0
\end{array}\right]\left[\begin{array}{c}
\mathrm{X}_{1 k}-\mathrm{X}_{2 k} \\
y_{k}
\end{array}\right]=0, \quad k=1, \cdots, 10
$$

where $\mathbf{X}_{1 k}=\left(x_{11 k}, x_{12 k}, x_{13 k}, x_{14 k}, x_{15 k}\right)^{\prime}$ and $\mathbf{X}_{2 k}=\left(x_{21 k}, x_{22 k}, x_{23 k}, x_{24 k}, x_{25 k}\right)^{\prime}$. Further-

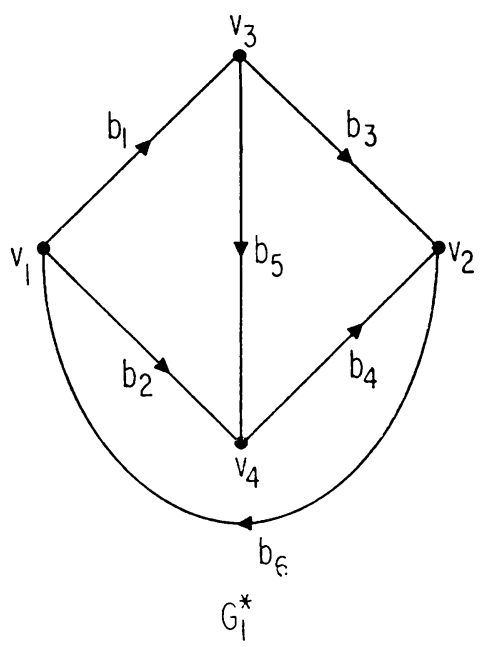

Figure 4. 
more, from the nature of the orientations of the branches of $G_{1}^{*}$, it should be obvious that for any optimal solution, $x_{21 k}=x_{22 k}=x_{23 k}=x_{24 k}=0$ for all $k$. Consequently, the flow constraints given in Eq. (A11) can be reduced to

$$
\begin{aligned}
x_{11 k}+x_{12 k} & =y_{k}, \\
x_{13 k}+x_{14 k} & =y_{k}, \quad k=1, \cdots, 10 \\
-x_{11 k}+x_{13 k}+x_{15 k}-x_{25 k} & =0,
\end{aligned}
$$

The remaining constraints of the synthesis procedure, specified by Eqs. (30c)-(33f), are

$$
\begin{gathered}
\mathbf{X}_{1 k}+\left[\begin{array}{c}
0 \\
0 \\
0 \\
0 \\
x_{25 k}
\end{array}\right] \leq\left[\begin{array}{l}
3 \\
3 \\
3 \\
3 \\
3
\end{array}\right]+\left[\begin{array}{l}
\Delta c_{1} \\
\Delta c_{2} \\
\Delta c_{3} \\
\Delta c_{4} \\
\Delta c_{5}
\end{array}\right]-F(k) \quad k=1, \cdots, 10, \\
\frac{1}{10} \sum_{k=1}^{10} y_{k}-3.0-.268 \tilde{z}^{1 / 2} \geq 0 \\
\hat{z}=z-\tilde{z}, \\
-\left(y_{1}, \cdots, y_{10}\right) W\left(y_{1}, \cdots, y_{10}\right)^{\prime}+z-\tilde{z} \geq 0,
\end{gathered}
$$

where $W$ is a $10 \times 10$ matrix with all main diagonal terms equal to 0.9 and all off diagonal terms equal to -0.1 .

The synthesis procedure can now be stated as: Find nonnegative numbers $\Delta c_{1}$, $\Delta c_{2}, \cdots, \Delta c_{5}, y_{1}, \cdots, y_{10}, x_{i j k}(i=1,2 ; j=1, \cdots, 5 ; k=1, \cdots, 10)$ such that $h_{1} \Delta c_{1}+\cdots,+h_{5} \Delta c_{5}$ is minimized subject to the constraints given in Eqs. (A12) and (A13a)-(A13d). This problem has a total of 123 variables and 83 constraints. Furthermore, only two of the constraints are nonlinear. If we fix $\hat{z}, \mathrm{Eq}$. (A13b) becomes linear in the remaining variables. We must now solve a concave programming problem with 122 variables, 82 linear constraints and one positive semidefinite quadratic constraint. Each solution for a given $\hat{z}$ is a minimum cost solution for the original problem when the sample variance is bounded. Therefore, by varying this parameter over a "reasonable" range, we can come arbitrarily close to the minimum cost problem with unspecified sample variance.

As a special case of the above problem, we will briefly consider the situation for which the sample variance $\hat{z}=0$. Under this condition, Eqs. (A13b) and (A13d) become

$$
\begin{gathered}
\frac{1}{10} \sum_{k=1}^{10} y_{k} \geq 3, \\
\left(y_{1}, \cdots, y_{10}\right) W\left(y_{1}, \cdots, y_{10}\right)^{\prime}=0 .
\end{gathered}
$$

Equation (A14a) is trivially linear. Furthermore, Eq. (A14b) can be replaced by a set of linear equations. It is well known that any quadratic form can be reduced to a sum of squares via a linear transformation of the form $\xi=\Gamma Y$. That is,

$$
\left(y_{1}, \cdots, y_{10}\right) W\left(y_{1}, \cdots, y_{10}\right)^{\prime}=\sum_{i=1}^{10} \alpha_{i} \xi_{i}^{2},
$$


where $\xi_{i}=\sum_{j=1}^{10} \alpha_{i j} y_{i}$ and the $\alpha_{i}$ 's and $\alpha_{i j}$ 's can be routinely found via the methods of Lagrange or Jacobi [10]. Furthermore, it can be shown that the rank of $W$ is nine and consequently exactly one $\alpha_{i}=0$ (say $\alpha_{10}$ ) while the remaining $\alpha_{i}$ 's are positive. Hence, Eq. (A14b) can be written as

$$
\sum_{i=1}^{9} \alpha_{i} \xi_{i}^{2}=0
$$

However, the only way this equation can hold is if $\xi_{1}=\xi_{2}=\cdots=\xi_{9}=0$. Therefore, we may replace Eq. (A16) (and thus Eq. (A14b)) by the system of linear equations

$$
\sum_{i=1}^{10} \alpha_{i} y_{i}=0 \quad i=1, \cdots, 9 .
$$

This, in turn, means that for $\hat{z}=0$, we must solve a routine linear program similar to the one solved in the preceding example. Thus, we can easily find an upper bound for the minimum cost.

\section{Bibliography}

[1] H. Frank and S. L. Hakimi, Probabilistic flows through a communication network, IEEE Trans. on Circuit Theory, Vol. CT-12, No. 3, pp. 413-414, Sept. 1965

[2] H. Frank and S. L. Hakimi, On the optimum synthesis of statistical communication nets-pseudoparametric techniques, J. Franklin Inst. 284, 407-467 (1967)

[3] H. Frank and S. L. Hakimi, Parametric analysis of statistical communication nets, Quart. Appl. Math. XXVI, 249-263 (1968)

[4] I. T. Frisch, Optimization of communication nets with switching, J. Franklin Intitute, 275, 405-430 (1963)

[5] G. Hadley, Nonlinear and dynamic programming, Addison-Wesley, Reading, Mass., 1964

[6] M. Abramowitz and L. A. Stegun (Editors), Handbook of mathematical functions, National Bureau of Standards, Applied Math. Series 55, 1964, p. 933

[7] N. Deo and S. L. Hakimi, Minimum cost increase of the terminal capacities of a communication net, IEEE Trans. on Comm. Tech., Vol. COM-14, No. 1, pp. 63-64, Feb. (1966)

[8] C. E. Clark, The greatest of a finite set of random variables, Operations Research, 9, 145-162 (1961)

[9] W. Feller, An introduction to probability theory and its applications, Vol. 1, Wiley, New York, 1957, pp. 177-178

[10] F. R. Gantmacher, Matrix theory, Vol. 1, Chelsea, New York, 1959, pp. 299-304 